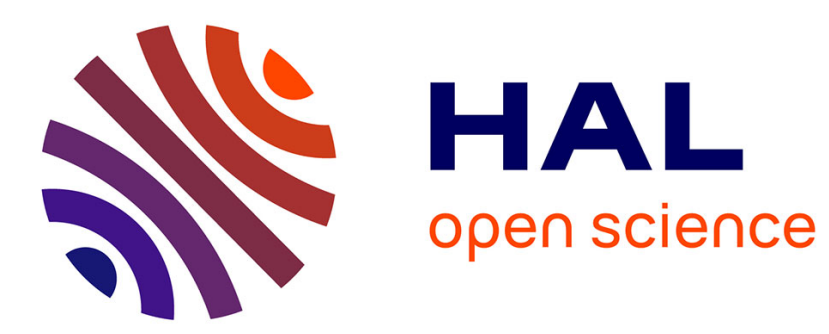

\title{
Effective connectivity in subcortical visual structures in de novo Patients with Parkinson's Disease
}

Emmanuelle Bellot, Louise Kauffmann, Véronique Coizet, Sara Meoni, Elena Moro, Michel Dojat

\section{- To cite this version:}

Emmanuelle Bellot, Louise Kauffmann, Véronique Coizet, Sara Meoni, Elena Moro, et al.. Effective connectivity in subcortical visual structures in de novo Patients with Parkinson's Disease. NeuroimageClinical, 2022, 33, pp.102906. 10.1016/j.nicl.2021.102906 . hal-03582724

HAL Id: hal-03582724

https://hal.univ-grenoble-alpes.fr/hal-03582724

Submitted on 21 Feb 2022

HAL is a multi-disciplinary open access archive for the deposit and dissemination of scientific research documents, whether they are published or not. The documents may come from teaching and research institutions in France or abroad, or from public or private research centers.
L'archive ouverte pluridisciplinaire HAL, est destinée au dépôt et à la diffusion de documents scientifiques de niveau recherche, publiés ou non, émanant des établissements d'enseignement et de recherche français ou étrangers, des laboratoires publics ou privés. 


\title{
Effective connectivity in subcortical visual structures in de novo Patients with Parkinson's Disease
}

\author{
Emmanuelle Bellot $^{\mathrm{a}}$, Louise Kauffmann ${ }^{\mathrm{b}}$, Véronique Coizet $^{\mathrm{a}}$, Sara Meoni ${ }^{\mathrm{a}, \mathrm{b}, \mathrm{c}}$, Elena Moro ${ }^{\mathrm{a}, \mathrm{b}}$, \\ Michel Dojat ${ }^{\mathrm{a} \text {,* }}$ \\ ${ }^{a}$ University Grenoble Alpes, Inserm U1216, Centre Hospitalier Universitaire de Grenoble, Grenoble Institute of Neurosciences, Grenoble, France \\ ${ }^{\mathrm{b}}$ Laboratory of Psychology and Neurocognition, CNRS UMR 5105, Grenoble, France \\ ${ }^{\mathrm{c}}$ Movement Disorders Unit, Division of Neurology, CHU Grenoble Alpes, Grenoble, France
}

\section{A R T I C L E I N F O}

\section{Keywords:}

Dynamic causal modeling

fMRI

Human vision

Neuroimaging

Superior colliculus

\begin{abstract}
A B S T R A C T
Background: Parkinson's disease (PD) manifests with the appearance of non-motor symptoms before motor symptoms onset. Among these, dysfunctioning visual structures have recently been reported to occur at early disease stages.

Objective: This study addresses effective connectivity in the visual network of PD patients.

Methods: Using functional MRI and dynamic causal modeling analysis, we evaluated the connectivity between the superior colliculus, the lateral geniculate nucleus and the primary visual area V1 in de novo untreated PD patients $(n=22)$. A subset of the PD patients $(n=8)$ was longitudinally assessed two times at two months and at six months after starting dopaminergic treatment. Results were compared to those of age-matched healthy controls $(\mathrm{n}=22)$.

Results: Our results indicate that the superior colliculus drives cerebral activity for luminance contrast processing both in healthy controls and untreated PD patients. The same effective connectivity was observed with neuromodulatory differences in terms of neuronal dynamic interactions. Our main findings were that the modulation induced by luminance contrast changes of the superior colliculus connectivity (self-connectivity and connectivity to the lateral geniculate nucleus) was inhibited in PD patients (effect of contrast: $p=0.79$ and $p=0.77$ respectively). The introduction of dopaminergic medication in a subset $(n=8)$ of the PD patients failed to restore the effective connectivity modulation observed in the healthy controls.

Interpretation: The deficits in luminance contrast processing in PD was associated with a deficiency in connectivity adjustment from the superior colliculus to the lateral geniculate nucleus and to V1. No differences in cerebral blood flow were observed between controls and PD patients suggesting that the deficiency was at the neuronal level. Administration of a dopaminergic treatment over six months was not able to normalize the observed alterations in inter-regional coupling. These findings highlight the presence of early dysfunctions in primary visual areas, which might be used as early markers of the disease.
\end{abstract}

\section{Introduction}

Parkinson's disease (PD) is a progressive neurodegenerative disorder affecting more than six million people worldwide (Collaborators, 2018). The onset of the pathological process and the so-called prodromal phase of PD is believed to start 10-20 years before the classical motor manifestations (tremor, rigidity and bradykinesia) (Mahlknecht et al., 2015).
Several non-motor symptoms develop during this prolonged prodromal phase (Siderowf and Lang, 2012), especially sensory dysfunctions like hyposmia (Lerner and Bagic, 2008; Siderowf et al., 2012) and oculovisual changes in pupil reactivity, color vision or visuo-motor adaptation (Armstrong, 2015). Several signs of a dysfunctioning visual pathway appear from early stages of PD (Weil et al., 2016) in the retina (Ahn et al., 2018) and progress along with the disease to higher visual

Abbreviations: BOLD, blood oxygen level-dependent; DCM, dynamic causal modeling; fMRI, functional magnetic resonance imaging; LGN, lateral geniculate nucleus; PD, Parkinson's disease; ROI, region of interest; SC, superior colliculus; V1, primary visual cortex.

* Corresponding author at: Grenoble Institut des Neurosciences, INSERM U1216, Bâtiment Edmond Safra, Chemin Fortuné Ferrini, 38700 La Tronche, France.

E-mail address: Michel.Dojat@inserm.fr (M. Dojat). 
brain areas, from V1 to the fusiform gyrus (Cardoso et al., 2010). PD patients show visuospatial and visuoperceptual deficits, which reflect structural changes in temporoparietal cortical regions (Pereira et al., 2009). Considering the systematic occurrence of neuron degeneration in subcortical structures before invasion of the cortex, alterations would be expected in brain regions involved in the first steps of visual processing such as the superior colliculus (SC) and the lateral geniculate nucleus (LGN); such changes may constitute early biomarkers of the disease.

Neuroimaging is a powerful tool for improving our understanding of both regional-specific dysfunction (Grafton et al., 1994) and brain connectivity changes in PD (Rowe, 2010). Several studies have explored resting-state functional connectivity (i.e. the statistical dependency of interregional activity) (Hacker et al., 2012; Helmich et al., 2010; Skidmore et al., 2011; van Eimeren et al., 2009; Wu et al., 2009). Fewer studies have examined changes in effective connectivity, i.e. how an activity network involving different brain regions is affected by a pathology, focusing on the detection of changes in cortical motor network coupling (Buijink et al., 2015; Dirkx et al., 2016; Marreiros et al., 2013; Nettersheim et al., 2019; Palmer et al., 2009; Rowe et al., 2002; Rowe et al., 2010) and modulatory actions of deep brain stimulation (Kahan et al., 2012; Kahan et al., 2014). To our knowledge, no study has been published concerning the subcortical and cortical effective connectivity in the visual network and how it is affected by PD.

Using functional MRI (fMRI) in healthy subjects of different ages, we showed an increase in the blood oxygen level dependent (BOLD) responses along the visual pathway, within the SC, the LGN and V1, in response to increasing luminance contrast in the range of $1-9 \%$, which changed with normal aging (see Fig. 1 for a schematic representation of the fMRI data) (Bellot et al., 2016). In a subsequent study performed in de novo $\mathrm{PD}$ patients, we found already present a functional deficit in both the SC and the LGN that was not compensated for by dopaminergic treatment at six months (see Supplementary Materiel, SM: Fig. S1) (Moro et al., 2020). The activation in our three regions of interest (ROIs) was correlated with luminance contrast modulation but did not inform us about the ROIs interactions. The main aim of the present study was to further investigate these findings by addressing brain connectivity, i.e., interactions between our visual ROIs, and exploring how visual information processing within this network might be already affected in de novo untreated PD patients. We hypothesized that the effective connectivity between the SC, the LGN and V1 would be altered in de novo PD patients compared to age-matched healthy controls and that the initiation of dopaminergic treatment over six months would not restore the

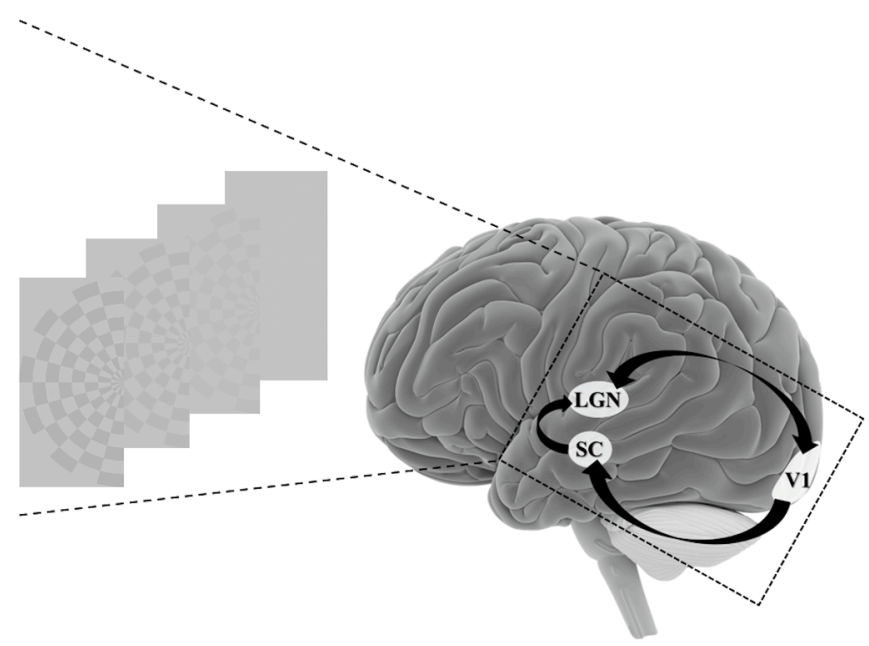

Fig. 1. Illustration of the visual stimulus and the three subcortical and cortical regions of interest involved in the first image processing steps and studied with fMRI. Black arrows indicate the anatomical connections based on the literature. $\mathrm{SC}=$ superior colliculus, LGN = lateral geniculate nucleus, $\mathrm{V} 1=$ primary visual area. inter-regional coupling. We analyzed effective connectivity using dynamic causal modeling (DCM), a probabilistic (Bayesian) modeling technique that estimates the coupling between selected brain regions based on fMRI data i.e., their BOLD responses to a specific stimulation, in our case visual stimuli. The present study is focused on the analysis of effective connectivity in PD patients and healthy controls based on the data published in (Bellot et al., 2016; Moro et al., 2020).

\section{Materials and methods}

\subsection{Participants}

Thirty healthy subjects (CT-30) and 22 de novo PD patients were recruited at the Movement Disorders Center of the Centre Hospitalier Universitaire de Grenoble (Grenoble, France). Inclusion criteria were: recent diagnosis of $\mathrm{PD}$ ( $<1$ year of motor symptoms' onset) according to the Movement Disorder Society (MDS) clinical diagnostic criteria of Parkinson's disease (Postuma et al., 2015), stage 1 or 2 of the Hoehn and Yahr scale (Hoehn and Yahr, 1967), with no psychiatric disorders, no antiparkinsonian treatment, absence of ophthalmological issues (cataract, retinal pathologies or glaucoma) and no contraindication to brain MRI (including relevant resting tremor). A subset of this control group, CT-22, was age-matched with the PD patients. Additionally, eight patients from the PD- $d n$ group, who had started L-dopa or dopamine agonist treatment after the first brain MRI study, were further evaluated twice: at six weeks to two months (session S1, resp. PD-2), and again at six months (session S2, resp. PD-6) after anti-PD treatment onset. All the selected participants were enrolled in our two previous studies (Bellot et al., 2016; Moro et al., 2020). All participants provided written informed consent to the study. The study was approved by the local ethics committee (ID RCB-2012-A00310-43 and ID RCB-2014-A0183542) and is registered at ClinicalTrial.gov (NCT02488395).

\section{2. $f M R I$ design and procedure}

The techniques used for visual stimuli and MRI acquisition have been described in detail elsewhere (Bellot et al., 2016). Briefly, subjects were presented in each visual hemi-field with a series of achromatic checkerboards, viewed via a mirror fixed on the head coil and flashing at a frequency of $4 \mathrm{~Hz}$ on a grey background, with four levels of luminance contrast (1, 3, 5 and 9\%) to avoid SC saturation (Schneider and Kastner, 2005). A block-design visual paradigm was used with each luminance contrast level presented in separate 12-s blocks. To ensure accurate activation localization in SC and limit partial volume effect, 32-channel SENSE coil imaging was performed at 3T, with a spatial resolution of 1.5 $\mathrm{mm}$ in each direction for EPI sequence and an acquisition volume centered on the SC structure. A high-resolution T1-weighted (MPRAGE) sequence and a T1-weighted FGATIR sequence were acquired to facilitate the manual delineation of SC and LGN (see details in SM). The cardiac signal was indirectly recorded at $100 \mathrm{~Hz}$ using finger photoplethysmography. Functional data analysis was performed using the single-participant general linear model (GLM) for block-designs with SPM12 (Wellcome Department of Imaging Neuroscience, London, U.K.) implemented in Matlab. The design matrix was constructed in order to remove possible motion components and cardio-respiratory effects, the main source of noise in the BOLD signal in the SC (see SM).

\subsection{Statistical analysis}

\subsubsection{Dynamic causal modeling for BOLD responses}

DCM (Friston et al., 2003; Penny et al., 2010) explores changes in the effective connectivity, i.e. how the influence exerted by one region over other regions can be affected by the experimental conditions. DCM allows inferences to be made on the neural mechanisms underlying the observations, in our study based on the BOLD time course in three ROIs (see SM for Time series extraction). Causal network models representing 
our hypotheses on these mechanisms were constructed and their parameters estimated (see SM about the specification of such models in DCM). Three sets of parameters were considered: the driving input parameter being visual stimulation in our models, the intrinsic connection parameters and the modulatory parameters that define how the effective connectivity may be modulated by, in our case, luminance contrast variations. In DCM, not all the intrinsic connections are necessarily affected by the modulation, leading to alternative plausible models that should be estimated.

The aim of this study was to assess whether differences observed in fMRI responses in PD patients compared to age-matched controls, could be explained by PD- and dopaminergic treatment- dependent alterations in the architecture of effective connectivity between the visual ROIs, and/or in the connectivity strength and its sensitivity to change by luminance contrast. To this end, the model best able to explain the data was first defined for each group (control, PD before and after dopamine treatment). Differences between groups in terms of the winning model was an indication of a change in connectivity architecture. The estimated parameters of each respective winning model were examined and compared across groups.

\subsubsection{Model space}

Six possible endogenous connections were considered between the three visual structures of interest, the LGN, SC and V1 (see Fig. 2, matrix A of the network). Neurophysiological studies in primates have shown bidirectional structural connections between the LGN and the V1 (Briggs and Usrey, 2011; Merigan and Maunsell, 1993; Sherman and Koch, 1986) as well as structural connections from the SC to the LGN (Benevento and Fallon, 1975; Harting et al., 1978; Munoz and Coe, 2011). Connections from the V1 to the SC have also been identified in macaque monkeys (Cerkevich et al., 2014; Künzle and Akert, 1977; Kuypers and Lawrence, 1967; Lock et al., 2003; Wurtz and Goldberg, 1972). Indeed, injections of anterograde tracers in non-human primates indicates that the SC receives input from most areas of visual cortex (Weller et al., 2002). However, to our knowledge, no direct inputs from the SC to the V1 or from the LGN to the SC have been reported so far. It should be noted that effective connectivity between two regions does not necessarily imply direct anatomical connections as the influence of one region over another can be mediated by other relay structures. For example, the SC and the V1 are connected via the pulvinar (Pennartz et al., 2019). The visual stimuli used in this study entered all models as a driving input to the two subcortical nodes, the LGN and the SC, both receiving direct visual inputs from the retina.

\subsubsection{Connectivity modulation}

A model space was designed by varying the subset of connections modulated by luminance contrast variations. This was based on our previous fMRI results (Bellot et al., 2016) showing that luminance contrast fluctuations modulated the activations in the visual ROIs (increased response with increasing contrast, see SM: Fig. S1). For the effective connectivity study, the lowest contrast condition (1\%) was considered to constitute a baseline from which the response was further modulated by contrast increase. Based on this notion, the remaining contrast conditions (3\%, 5\% and $9 \%$ ) were used as modulating inputs, to explore the connectivity modulation with increasing contrasts relative to the $1 \%$ contrast condition. These transient perturbations were implemented as bilinear terms assuming a bilinear evolution based on our previous results showing a linear relation between BOLD signal and luminance contrast in the three regions within the variation range used (Bellot et al., 2016). A series of seven models was considered to test the modulatory influence of luminance contrast on effective connectivity from LGN, V1 or SC, to the two other structures (V1 and SC, LGN and SC, or LGN and V1, cases 1 to 3 respectively), or from V1 + LGN, V1 + SC or $\mathrm{LGN}+\mathrm{SC}$ to the SC, LGN or V1, respectively (cases 4 to 6 respectively), as well as all the possible modulations (case 7). Also considered were models with the same structure and modulations as models 1 to 7 but with an additional self-modulation of the SC, leading to a total of fourteen models to compare (see Fig. 2). These models ( 8 to 14) were used to account for the possibility of a dysfunction of the SC self-modulation in response to luminance contrast in PD. All 14 models were fed

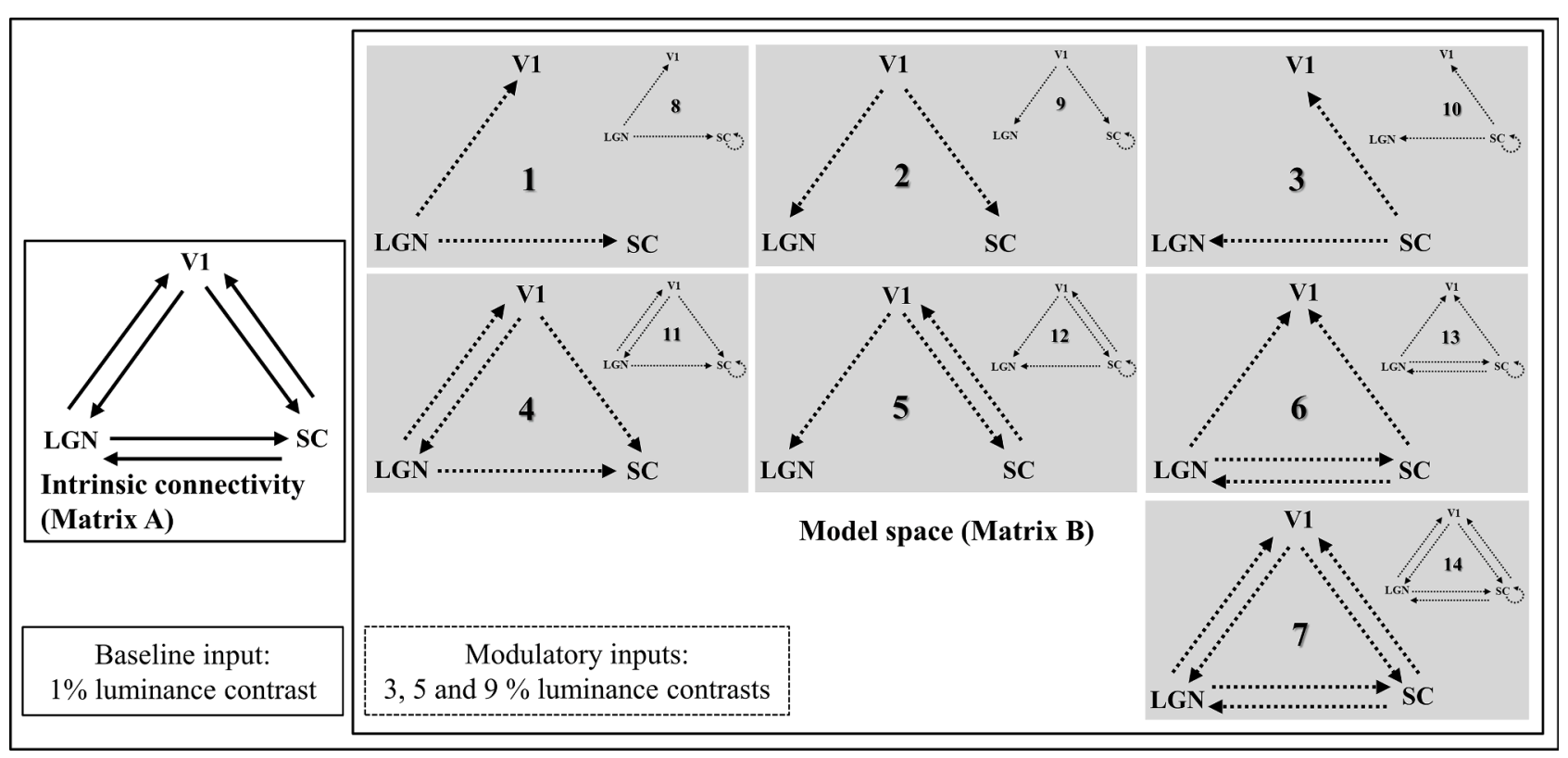

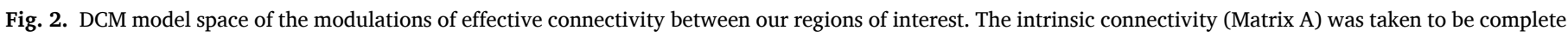

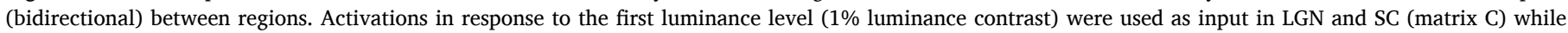

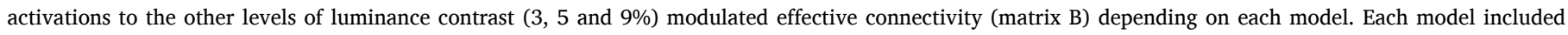

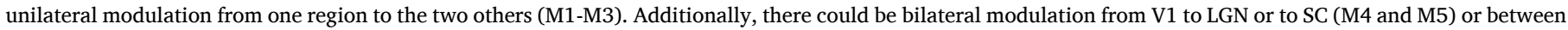

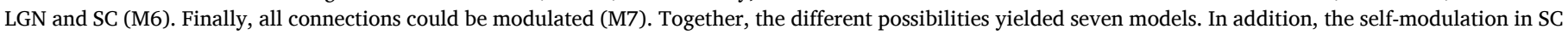

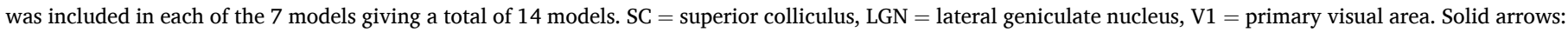
intrinsic connections. Dashed arrows: connections modulated by luminance. 
successively with the functional datasets from four groups: control subjects and untreated PD patients or treated PD patients with dopaminergic treatment (2- or 6-months uptake). Model selection and inference on parameters of the best model were made. For group-level inference on model structure, we then considered a Random-effects (RFX) BMS, taking into account pathophysiological variations across patients (Stephan et al., 2010) (see SM).

\subsection{Data availability}

MR data supporting the results of this study are available from the corresponding author, on a collaborative basis.

\section{Results}

\subsection{Demography}

Details of the clinical characteristics of PD patients are shown in Table 1. There were no significant differences between the CT-22 (9 women, age $55.5 \mathrm{y} . \pm 9.4$ ) and PD- $d n$ groups of patients (5 women, age $57.3 \mathrm{y} . \pm 10.5$ ), in terms of age (two-sample $t$-test; $\mathrm{p}=0.57$ ) and gender $(\mathrm{p}=0.2)$. The subset of the PD population ( $\mathrm{n}=8,3$ women, age 55,62 $\mathrm{y}$. \pm 7.3 ) with the follow-up at 2 and 6 months did not differ in terms of age (two sample $t$-test, $\mathrm{p}=0.75$ ) and gender $(\mathrm{p}=0.53)$.

\subsection{Effective connectivity: Healthy controls and PD patients}

\subsubsection{The best effective connectivity model for controls}

In the control group (CT-22), the exceedance probabilities (xp) for the 14 models are shown in Fig. 3A. As can be seen in this Figure, Model 10, which emphasizes the role of the SC, outperformed all competing models $(x p=0.93)$. It was characterized by self-modulation by luminance contrast in the SC and unilateral modulation from the SC to the LGN and the V1. The fitting quality of this model was indicated for one subject (see SM: Fig. S2). Its parameters are reported in SM: Table 1. Additional results on the effect of age on the intrinsic connectivity in healthy controls (CT-30) are provided in SM (see SM: Fig. S3 and Table 2).

\subsubsection{The best effective connectivity model for PD patients}

As for the control group, the winning model for de novo (untreated)
PD patients (PD- $d n$ ) was model 10 (see Fig. $3 \mathrm{~A}, \mathrm{xp}=0.37$ ). However, the exceedance probability was lower in these patients $(x p=0.37)$ compared to controls ( $x p \geq 0.70$ ). The significance of the different parameters of the model is reported in SM: Table 1.

\subsubsection{Effective connectivity in $P D$}

The finding that the best-fitting model was the same for the PD patient and control groups, suggests that PD does not impact the architecture of connectivity between the three ROIs. We therefore examined the connectivity parameters on this model for both groups. As for controls, all intrinsic connectivity parameters were positive, i.e. an increase in activity in one region resulted in an increase in each target region (see Fig. 3B). PD- $d n$ patients' intrinsic connectivity parameters did not significantly differ from controls, as confirmed by the 2x6 ANOVA with Group (Controls CT-22, age-matched with PD patients- vs. PD-dn patients) as the between-subjects factor and Connection as within-subjects factor; this revealed a significant effect of Connection $(F(5,210)=5.82$, $\left.p<10^{-4}, \eta_{\mathrm{p}}{ }^{2}=0.23\right)$ but no significant effect of Group $(p=0.72)$ or interaction between Group and Connection $(p=0.89)$.

\subsubsection{Effect of luminance contrast modulation on the effective connectivity}

PD was revealed as a factor that significantly affected the SC selfmodulation $\left(F(1,42)=14.03, p<10^{-3}, \eta_{\mathrm{p}}^{2}=0.40\right)$ and the modulation of the SC to LGN connection $\left(F(1,42)=8.46, p<10^{-2}, \eta_{\mathrm{p}}{ }^{2}=0.29\right)$ but not the modulation of the SC to V1 connection $(p=0.15)$. Moreover, luminance contrast was another factor significantly affecting these three intrinsic connections (SC $\leftrightarrow$ SC self-modulation: $F(3,126)=8.08, p<10^{-}$ ${ }^{4}, \eta_{\mathrm{p}}{ }^{2}=0.28 ; \mathrm{SC} \rightarrow \operatorname{LGN}: F(3,126)=3.78, p<10^{-2}, \eta \mathrm{p}^{2}=0.15$; SC $\rightarrow \mathrm{V} 1:$ $\left.F(3,126)=6.07, p<10^{-3}, \eta_{\mathrm{p}}{ }^{2}=0.22\right)$. Interaction between Group and Contrast factors was only significant for the self-modulation of the SC ( $F$ $(3,126)=6.28, p<10^{-3}, \eta_{\mathrm{p}}{ }^{2}=0.23$; SC $\rightarrow$ LGN: $p=0.14$; SC $\rightarrow \mathrm{V} 1: p=$ 0.11) ( $2 \times 4$ ANOVA with Group, i.e. Controls $v s$. PD-dn patients, as between-subjects factor and Contrast as within-subjects factor, performed for each connection). To further investigate the effect of the modulation of the connections in PD, an ANOVA with Contrast as repeated-measure was performed for each connection. These analyses revealed a much-reduced weak effect of the luminance contrast modulation on the SC connections in PD-dn patients compared to controls (see Fig. 4). Indeed, the pathology (PD) appeared to have hampered the selfmodulation of SC (effect of contrast: $p=0.79$ ) and the modulation of the SC to LGN connection (effect of contrast: $p=0.77$ ). However, the

Table 1

Clinical characteristics of PD patients. (this table is similar to Table 1 in (Moro et al., 2020)).

\begin{tabular}{|c|c|c|c|c|c|c|c|}
\hline Patient & SEX & AgE, yr & H\&Y Scale stage & Year of Diagnosis & Disease Onset (Body side) & UPDRS & Treatment at 2 and $6 \mathrm{mo}$ \\
\hline 1 & M & 67 & 1 & 2016 & Left & 18 & NA \\
\hline 2 & $\mathrm{~F}$ & 45 & 1 & 2017 & Left & 11 & L-dopa/benserazide \\
\hline 3 & M & 61 & 1 & 2017 & Left & 31 & NA \\
\hline 4 & M & 59 & 1 & 2016 & Left & 11 & Pramipexole \\
\hline 5 & M & 68 & 1 & 2016 & Right & 5 & NA \\
\hline 6 & M & 76 & 1 & 2016 & Left & 17 & NA \\
\hline 7 & M & 58 & 1 & 2015 & Left & 23 & Rasagiline/pramipexole \\
\hline 8 & M & 59 & 1 & 2016 & Right & 15 & Rasagiline/pramipexole \\
\hline 9 & $\mathrm{~F}$ & 49 & 1 & 2014 & Left & 15 & Rasagiline/L-dopa Benserazide \\
\hline 10 & M & 36 & 1 & 2016 & Left & 9 & NA \\
\hline 11 & M & 47 & 2 & 2016 & Right & 11 & NA \\
\hline 12 & $\mathrm{~F}$ & 68 & 2 & 2014 & Right & 40 & Rasagiline/L-dopa benserazide \\
\hline 13 & M & 72 & 2 & 2017 & Left & 23 & NA \\
\hline 14 & M & 45 & 2 & 2017 & Right & 20 & NA \\
\hline 15 & M & 68 & 2 & 2017 & Right & 25 & NA \\
\hline 16 & M & 50 & 2 & 2015 & Right & 28 & L-dopa/triehyphenidyl \\
\hline 17 & M & 52 & 2 & 2016 & Left & 21 & NA \\
\hline 18 & $\mathrm{~F}$ & 56 & 2 & 2016 & Left & 24 & NA \\
\hline 19 & M & 67 & 2 & 2015 & Right & 14 & NA \\
\hline 20 & M & 57 & 2 & 2014 & Right & 18 & Rasagiline/ropinirole \\
\hline 21 & M & 44 & 2 & 2016 & Right & 27 & NA \\
\hline 22 & $\mathrm{~F}$ & 56 & 2 & 2016 & Right & 21 & NA \\
\hline
\end{tabular}

$\mathrm{F}=$ female; $\mathrm{M}=$ male; H\&Y: Hoehn and Yahr; NA = not applicable; PD = Parkinson's disease; UPDRS = Unified PD Rating Scale. 
A.

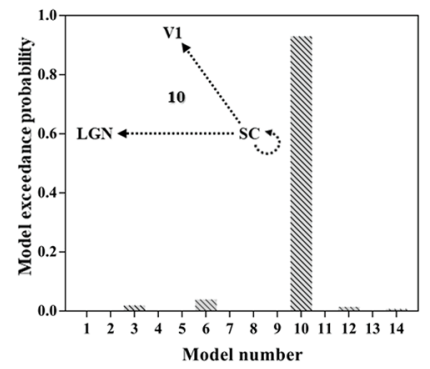

B.

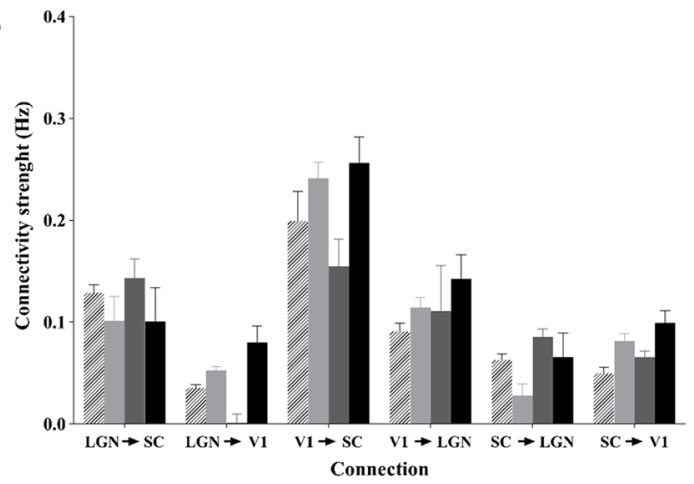

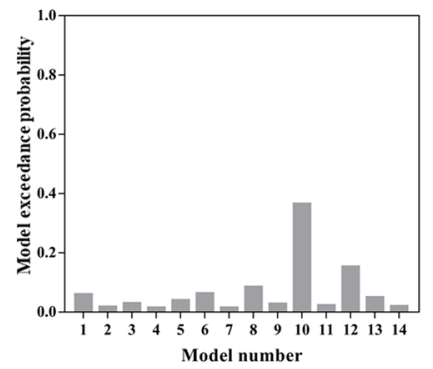
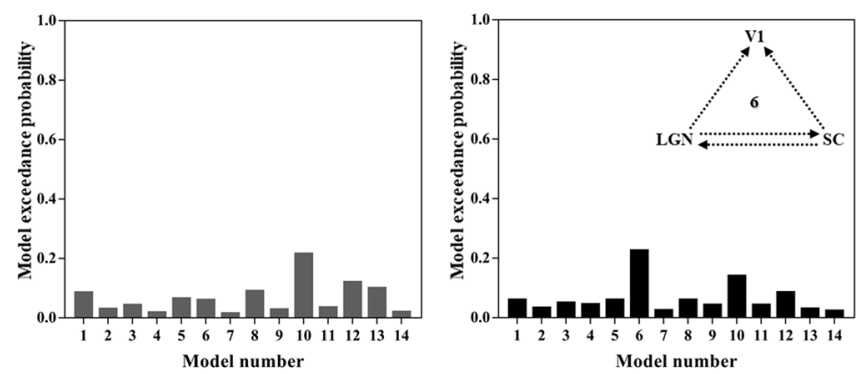

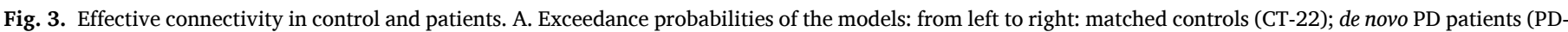

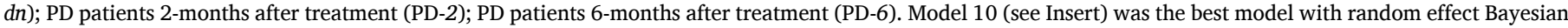

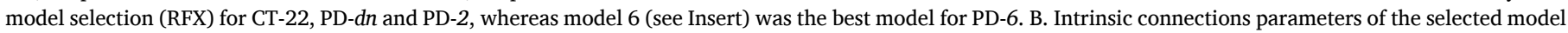

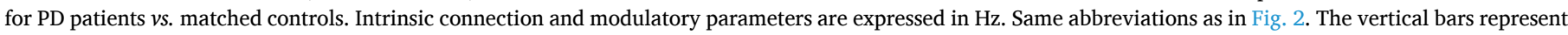
standard errors.
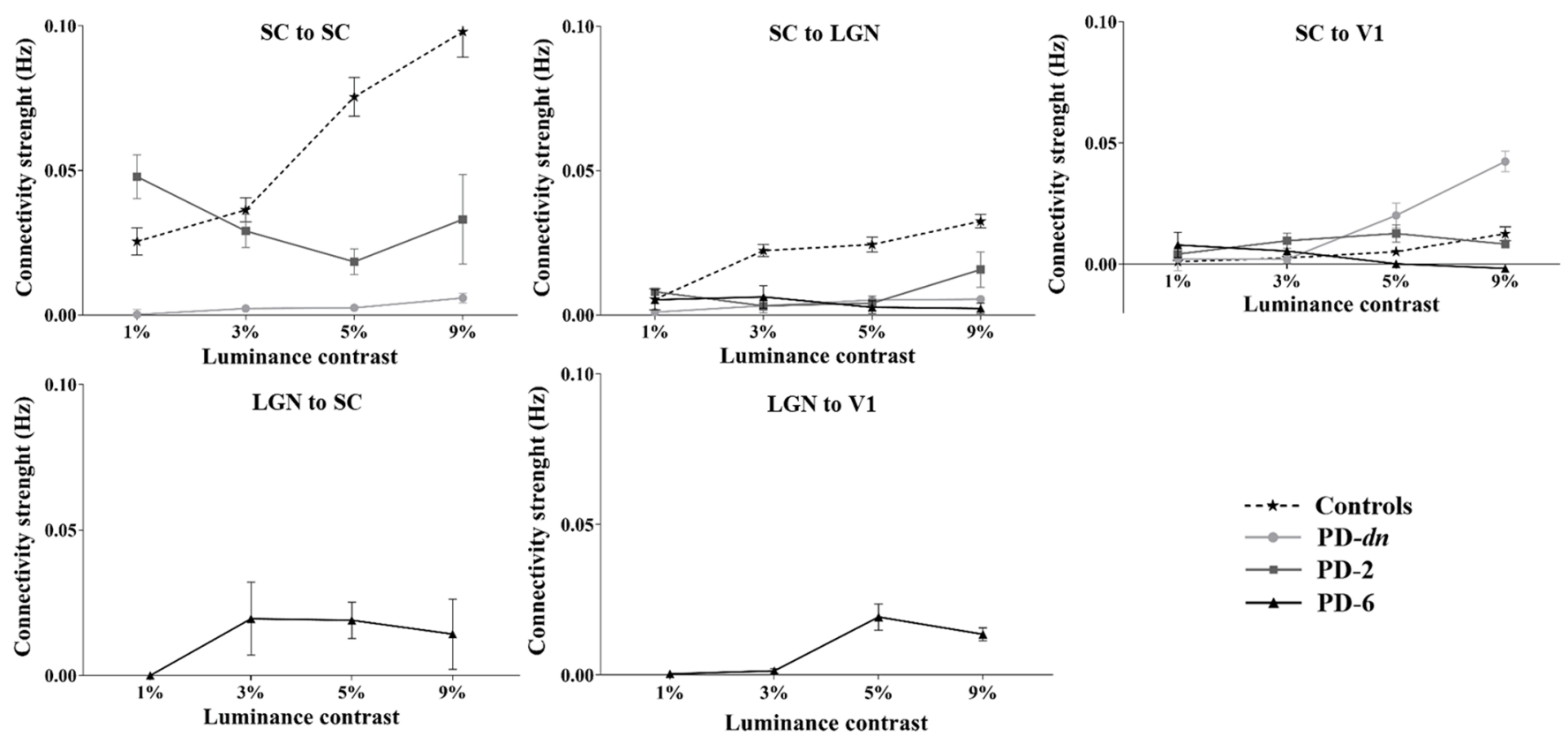

Fig. 4. Intrinsic connection modulation with luminance contrast. Each diagram corresponds to connections present in model 10 and model 6 and shows the corresponding modulation of the connectivity strength (expressed in $\mathrm{Hz}$ ) by luminance contrast variations for Controls (CT-22), PD patients without treatment (PD-dn) and with 2 (PD-2) or 6 (PD-6) months of dopaminergic treatment. Dashed line and stars: CT-22. Bold line: PD patients; Circle: PD-dn, Square: PD-2, Triangle: PD-6. Intrinsic connection and modulatory parameters are expressed in Hz. Same abbreviations as in Fig. 2. The vertical bars represent standard errors.

luminance contrast significantly modulated the SC to V1 connection ( $F$ $\left.(3,63)=3.66, p=0.02, \eta_{\mathrm{p}}^{2}=0.29\right)$ in PD-dn patients (see Fig. 4).

\subsection{Effective connectivity in PD: Medication effects}

A subset of the PD participants $(\mathrm{n}=8)$ was assessed longitudinally after two (PD-2) and six (PD-6) months of dopaminergic treatment.

\subsubsection{The best effective connectivity model}

For PD-2, the same model (Model 10) was selected ( $x p=0.22$ ) (see Fig. 3A). However, a different model, Model 6, was selected for PD-6 (xp $=0.23$ ) (see Fig. $3 \mathrm{~A}$ right). The SC plays a less central role in model 6 compared to in model 10. This suggests a reduced response of SC following six months of treatment. On the other hand, LGN is more implicated in model 6 with additional connections to the V1 and to the 
SC. The significance of the different parameters of these models ( 6 and 10) is reported in SM: Table 1.

\subsubsection{Effect of the dopaminergic treatment on the effective connectivity}

At two and six months of treatment, intrinsic connectivity parameters from models 6 and 10 were positive (see Fig. 3B). To test the effect of dopaminergic treatment on these parameters, a 3x6 ANOVA with Group (PD-dn vs. PD-2 vs. PD-6) as between-subjects factor and Connection as within-subjects factor was performed. Connection $(F(5,175)=6.56, p=$ $10^{-4}, \eta_{\mathrm{p}}{ }^{2}=0.25$ ) was revealed as a significant factor but not Group (i.e. treatment) $(p=0.43)$, and no interaction between Group and Connection $(p=0.75)$ was observed (see Fig. 3B).

\subsubsection{Effects of the dopaminergic treatment on the modulation of the effective connectivity by luminance contrast}

At two months of treatment, only the modulation by luminance contrast of the connection SC $\leftrightarrow$ SC was significantly affected by the medication $\left(F(1,28)=6.87, p=0.02, \eta_{\mathrm{p}}{ }^{2}=0.31\right.$; SC $\rightarrow$ LGN: $p=0.48$; $\mathrm{SC} \rightarrow \mathrm{V} 1: p=0.46)$. Contrast had no significant effect on the three intrinsic connections (SC $\leftrightarrow$ SC: $p=0.69$; SC $\rightarrow \mathrm{LGN}: p=0.43$; SC $\rightarrow \mathrm{V} 1$ : $p=0.07$ ) and no interaction was found between Group and Contrast (SC $\leftrightarrow$ SC: $p=0.59$; SC $\rightarrow$ LGN: $p=0.59$; SC $\rightarrow$ V1: $p=0.12)(2 \times 4$ ANOVA with Group, i.e. PD- $d n v s$. PD-2, as between-subjects factor and Contrast as within-subject factor, performed for each connection) (see Fig. 4).

After six months of treatment, as characterized by the selected model 6 , four connections seemed modulated by luminance contrast, i.e. SC $\rightarrow$ $\mathrm{LGN}, \mathrm{SC} \rightarrow \mathrm{V} 1, \mathrm{LGN} \rightarrow \mathrm{SC}$ and LGN $\rightarrow \mathrm{V} 1$. A 2x4 ANOVA was performed for each of the two common connections of models 6 and 10 (SC $\rightarrow$ LGN and SC $\rightarrow$ V1) with Group (PD- $d n v s$. PD-6) as between-subjects factor and Contrast as within-subjects factor. Neither Group (SC $\rightarrow$ LGN: $p=$ 0.91 ; SC $\rightarrow \mathrm{V} 1: p=0.24$ ) nor Contrast (SC $\rightarrow$ LGN: $p=0.98$; SC $\rightarrow$ V1: $p$ $=0.32$ ) as factors showed any significant effect on these connections. An interaction was found between Group and Contrast for connection SC $\rightarrow$ V1 $\left(F(3,84)=2.87, p=0.04, \eta_{\mathrm{p}}{ }^{2}=0.17\right.$; SC $\rightarrow$ LGN: $\left.p=0.80\right)$, showing a significant difference between the two groups at $9 \%$ contrast $(p=$ 0.01) (see Fig. 4).

\section{Discussion}

This is the first study addressing the effective connectivity in the visual network of de novo PD patients. By testing competing models of connectivity, we have demonstrated that the SC drives cerebral activity in the visual network for luminance contrast processing, both in healthy controls and PD patients off medication. Our results reveal that the corresponding deficit in contrast processing reported early in PD patients seems related to a deficiency in connectivity adjustment from the SC to both the LGN and the primary visual area V1. Dopaminergic treatment over six months is not sufficient to normalize this abnormal connectivity. The DCM model space we considered is based on our previous works demonstrating: 1) the three regions of interest SC, LGN and V1 exhibited a BOLD response modulated by luminance contrast in the healthy population; (Bellot et al., 2016) and 2) the functioning of SC was altered in de novo PD patients (Moro et al., 2020). This latter study motivated our introducing models of connectivity that included the selfmodulation of the SC.

Pivotal role of the SC for luminance contrast processing. For healthy subjects, the DCM analysis supports a model with a high excellence probability $(\mathrm{xp}=0.93)$ where luminance contrast modulated the SC selfconnectivity, as well as effective connectivity from the SC to both the V1 and the LGN. For these subjects, an increase in luminance contrast resulted in an increase of the SC self-connectivity for all age ranges (except the elderly) and an increase of the SC to LGN and V1 couplings (see SM: Fig. S4C and SM: Table 2). Our data also indicate that connectivity strength within and from the SC decreases with normal aging (see SM: Fig. S4B and SM: Table 2). This decrease may explain the agerelated luminance contrast sensitivity loss observed in these regions with BOLD measurements and also based on behavioral responses (Bellot et al., 2016). Due to the small size of our regions of interest, and in order to maximize the signal-to-noise ratio, we pooled the right and left hemisphere data for each ROI for each subject. Indeed, for the SC that plays a central role in our study, visual information from the right to the left colliculus can be transferred via the inter-collicular commissure or other inter-hemispheric tracts (Georgy et al., 2016).

Effect of $P D$ on effective connectivity. We addressed the nonmotor impact of PD and studied the effective connectivity of the visual system as measured by DCM, based on fMRI measurements. In fMRI, the measured BOLD signal reflects changes in hemodynamic parameters induced by neural activation and is considered as an index of cerebral activity. However, aging, pathological states or drugs may influence hemodynamics and alter the neurovascular coupling. We have previously shown that observed BOLD signal variations both in healthy subjects (Bellot et al., 2016) and in PD patients (Moro et al., 2020) were due to neural activity changes and not a consequence of perfusion alterations. We measured CBF using the ASL technique to verify that the basal brain perfusion level was similar between the two groups in each region of interest and under anti-PD treatment. Thus, BOLD changes reflected neural activity changes and were not a consequence of a neurovascular coupling deficiency.

The present study has investigated the alterations in visual processing due to PD at the first stages, both at the cortical level in the V1 and at the subcortical level in the LGN and the SC. Metabolic differences can be present in PD patients compared to controls that remain unobserved by the MRI techniques we used. The observed BOLD signal modulation due to luminance changes and correlated to visual perception was similar in PD patients and healthy controls in V1 suggesting corresponding neural activity changes at the cortical level. Then, it is unlikely that the observed differences in the two other structures SC and LGN were not due to changes in brain activity but to metabolic alterations only present at this subcortical level.

The SC is a complex sensory-motor brainstem structure that acts as a sentinel in detecting sudden environmental changes and responding to these changes. It integrates multimodal sensory information from visual, auditory, and tactile sources; generates outputs for gaze, head, and arm movement; and sends priority signals to the substantia nigra pars compacta and the intralaminar nucleus of the thalamus. Abnormal responses of SC to visual stimuli have been demonstrated in a PD rat model (Rolland et al., 2013) and more recently in humans (Moro et al., 2020). In a post-mortem study (Erskine et al., 2017), neurodegenerative changes in the SC tissue were found in Lewy bodies dementia that may contribute to visual attention deficits and visual hallucinations; manifestations which are also present in PD patients (Diederich et al., 2009; Frei and Truong, 2017).

Our DCM analysis revealed that the best model explaining the data in terms of intrinsic and modulated connectivity was the same for healthy controls and de novo untreated PD patients (see Fig. 3A). In concordance with previous studies, this model emphasized the pivotal role of the SC for luminance contrast processing. However, we found that the modulation of the SC connectivity (self-connectivity and connectivity to the LGN) by luminance contrast was inhibited in these PD patients (see SM: Table 1). This suggests that while PD patients have the same effective connectivity as healthy controls, the neuromodulation is different in terms of neuronal dynamic interactions. The SC-V1 connection modulation by luminance contrast was preserved at this early stage of $\mathrm{PD}$, thus explaining why de novo PD patients did not report about visual deficits.

The best-fitting models for PD patients and for healthy controls were identical but the exceedance probability was rather low for the former $(\mathrm{xp}=0.37)$ compared to the latter $(\mathrm{xp}=0.93)$. A methodological alternative would have been to consider Bayesian Model Averaging. In this method, the parameters of all plausible models are averaged while taking into account the respective weight for each model (Stephan et al., 2010). This method also allows to compare the differences between models. Although it could have been useful to compare differences 
between models 6 and 10 after 6-months treatment of PD patients, this alternative would also have rendered the inter-group comparison (i.e. healthy controls $v s$ PD patients), which was the main focus of the study, more difficult to interpret. We thus chose to identify the model which best fitted the observed data in healthy controls and revealed the effect of the pathology (and that of the drug, see below) on the same "best" model. In accordance with that previously observed (Moro et al., 2020), the decrease in $\mathrm{xp}$ for the best-fitting PD model reflects that luminance processing becomes dysfunctional in PD patients likely due to a dysfunctioning SC, the central structure in this model. The decrease in xp could also be due to the greater inter-subject variability in the BOLD signal for the PD group compared to the control group (Moro et al., 2020) and to possible metabolic alterations that affect PD patients (Sonninen et al., 2020).

Effect of $P D$ treatment. For brain regions involved in controlling movement, some studies (Nettersheim et al., 2019; Palmer et al., 2009) showed that dopaminergic medication normalized to some extent effective connectivity in PD patients. Similarly, a relative normalization of visuo-parietal connectivity in PD patients with dopaminergic medication during cued and uncued writing was found (Nackaerts et al., 2018). The results from our present study show that dopaminergic treatment, whether taken during 2 or 6 months, did not permit the recovery from abnormal SC connectivity to other brain regions. Findings from our analyses do however suggest that after six months of anti-PD treatment, the LGN plays a more important role (see model 6, Fig. 3A) in the investigated network, despite no evidence of recovery of the effective connectivity modulation by luminance contrast (see Fig. 4). This is consistent with the reduced response in the SC conjointly with an increased response of the LGN to luminance contrast after six month of treatment as observed in (Moro et al., 2020).

It has been suggested (Rolland et al., 2013) that the alteration of the SC response may occur as a compensatory mechanism in response to inhibition exerted by the loss of dopaminergic neurons in the substantia nigra reticulata. Therefore, dopaminergic treatment may not be adapted to rapidly (after 6 months administration) reverse this process (Palmer et al., 2009). Additionally, brain regions are part of complex internetwork interactions (Furl, 2015) suggesting that the anti-PD treatments may have, at this stage of the pathology, no effect on the functional responses of networks interacting and influencing the visual regions studied.

Our study has several limitations. Although the reasonable power for DCM analysis was reached (Goulden et al., 2010), the present study remains limited by the small size of the subject samples. Indeed, the number of patients on which we could assess the effect of medication on inter-regional coupling was only half that of the initial patient sample size. Our results should thus be considered as exploratory and require replication on a larger population. Furthermore, we did not consider a hemispheric difference in effective connectivity that could be induced by the lateralization of the disease. The BOLD signal used in the present study provides indirect neuronal information based on which connectivity models could be defined at a systems level using DCM but which hampers the interpretation of effective connectivity in terms of inhibition or excitation at the neuronal level. The poor temporal resolution of the BOLD response did not allow the assessment of temporal dynamics of the neuronal model. Based on the literature and our previous results both in humans and PD animal models, we consider the colliculus as a key sensory structure impacted at early stages of PD. Recently, we demonstrated alterations in the function of the superior SC, involved in visual information processing, at early stages of PD. The main goal of the present study was to investigate the role of SC in regional coupling both in normal and pathological conditions. To this end, we introduced models with or without SC self-modulation. Introduction of more models, for instance with self-modulation on the other ROIs, could be explored in future studies, although the robustness of the results obtained would diminish with increasing number of tested connections. Finally, considering the decreasing robustness of DCM analyses with increasing number of ROIs, we restricted our hypothesis testing to a small number of ROI, namely SC, LGN and V1, which we considered the most informative. It should be noted that the missing regions do not affect the estimates in the ROIs because influences from outside the restricted network are modelled as endogenous fluctuations (Daunizeau et al., 2011; Stephan et al., 2010). Nevertheless, we left several other activated regions unexplored. For example, neuroanatomical studies suggest that the SC forms a subcortical pathway that bypasses the striate cortex V1 and projects to the amygdala via the pulvinar. While the role of Pulvinar remains elusive (Fiebelkorn and Kastner, 2019), it is clearly a region of interest, at least in its inferior and ventrolateral parts, when studying vision in PD. The recent availability of digital atlases of the human thalamic nuclei (for instance (Najdenovska et al., 2018) would facilitate its automatic delineation and then its functional exploration. However, the functional heterogeneity of the pulvinar (Guedy and Vuilleumier, 2020) and its proximity to the choroidal artery could hamper the detection on the Bold modulation induced by luminance contrast variations, at least at $3 \mathrm{~T}$. Further research concerning the impact of the SC alterations on connectivity with these structures may help finely tune our understanding of the effects of the disease.

In summary, these findings provide useful insights into brain alterations occurring at early stages of PD and in particular highlight the presence of early dysfunctions in primary visual areas, which might be used as early markers of the disease.

Clearly, the measurement of such dysfunctions using an fMRI paradigm, as proposed in the present study, does not fit with a routine clinical evaluation. We currently explore the use of a specific intrasaccadic motion paradigm where the magnocellular pathway (including SC and LGN) is mainly involved (Nicolas et al., JOV 2021). The rationale is that the motion detection performance measured in the psycho-physical experiment using such a paradigm, would be poorer in $\mathrm{PD}$ patients compared to age-matched healthy controls due to the functional deficiency of the SC and the LGN.

\section{CRediT authorship contribution statement}

Emmanuelle Bellot: Investigation, Data curation, Formal analysis, Writing - review \& editing. Louise Kauffmann: Methodology, Formal analysis, Writing - review \& editing. Véronique Coizet: Conceptualization, Project administration, Writing - review \& editing. Sara Meoni: Resources, Writing - review \& editing. Elena Moro: Supervision, Funding acquisition, Writing - review \& editing. Michel Dojat: Conceptualization, Methodology, Funding acquisition, Supervision, Writing - original draft, Writing - review \& editing.

\section{Declaration of Competing Interest}

The authors declare that they have no known competing financial interests or personal relationships that could have appeared to influence the work reported in this paper.

\section{Acknowledgements}

The authors are grateful to the patients and to the healthy control group of individuals who took part in this study.

\section{Funding sources}

Emmanuelle Bellot is the recipient of a grant from the France Parkinson Foundation (France) and from the Université Grenoble Alpes. This work was partly supported by a grant from 'La Fondation de l'Avenir' (France). The Grenoble MRI facility IRMaGe is partly funded by the French program 'Investissement d'Avenir' run by the Agence Nationale pour la Recherche (ANR-11-INBS-0006). 


\section{Appendix A. Supplementary data}

Supplementary data to this article can be found online at https://doi. org/10.1016/j.nicl.2021.102906.

\section{References}

Ahn, J., Lee, J.-Y., Kim, T.W., Yoon, E.J., Oh, S., Kim, Y.K., Kim, J.-M., Woo, S.J., Kim, K. W., Jeon, B., 2018. Retinal thinning associates with nigral dopaminergic loss in de novo Parkinson disease. Neurology 91 (11), e1003-e1012.

Armstrong, R.A., 2015. Oculo-Visual Dysfunction in Parkinson's Disease. J. Parkinsons Dis. 5 (4), 715-726.

Bellot, E., Coizet, V., Warnking, J., Knoblauch, K., Moro, E., Dojat, M., 2016. Effects of aging on low luminance contrast processing in humans. Neuroimage 139, 415-426.

Benevento, L.A., Fallon, J.H., 1975. The ascending projections of the superior colliculus in the rhesus monkey (Macaca mulatta). J. Comp. Neurol. 160 (3), 339-361.

Briggs, F., Usrey, W.M., 2011. Corticogeniculate feedback and visual processing in the primate. J. Physiol. 589 (1), 33-40.

Buijink, A.W.G., van der Stouwe, A.M.M., Broersma, M., Sharifi, S., Groot, P.F.C., Speelman, J.D., Maurits, N.M., van Rootselaar, A.-F., 2015. Motor network disruption in essential tremor: a functional and effective connectivity study. Brain 138 (10), 2934-2947.

Cardoso, E.F., Fregni, F., Maia, F.M., Melo, L.M., Sato, J.R., Cruz, A.C., Bianchi, E.T., Fernandes, D.B., Monteiro, M.L.R., Barbosa, E.R., Amaro, E., 2010. Abnormal visual activation in Parkinson's disease patients. Mov. Disord. 25 (11), 1590-1596.

Cerkevich, C.M., Lyon, D.C., Balaram, P., Kaas, J.H., 2014. Distribution of cortical neurons projecting to the superior colliculus in macaque monkeys. Eye Brain 2014, 121-137.

Collaborators, G.P.s.D., 2018. Global, regional, and national burden of Parkinson's disease, 1990-2016: a systematic analysis for the Global Burden of Disease Study 2016. Lancet Neurol. 17, 939-953.

Daunizeau, J., David, O., Stephan, K.E., 2011. Dynamic causal modelling: a critical review of the biophysical and statistical foundations. Neuroimage 58 (2), 312-322.

Diederich, Nico J., Fénelon, Gilles, Stebbins, Glenn, Goetz, Christopher G., 2009. Hallucinations in Parkinson disease. Nat. Rev. Neurol. 5 (6), 331-342.

Dirkx, Michiel F., den Ouden, Hanneke, Aarts, Esther, Timmer, Monique, Bloem, Bastiaan R., Toni, Ivan, Helmich, Rick C., 2016. The Cerebral Network of Parkinson's Tremor: An Effective Connectivity fMRI Study. J. Neurosci. 36 (19), 5362-5372.

Erskine, D., Thomas, A.J., Taylor, J.P., Savage, M.A., Attems, J., McKeith, I.G., Morris, C. M., Khundakar, A.A., 2017. Neuronal loss and alpha-synuclein pathology in the superior colliculus and its relationship to visual hallucinations in Dementia with Lewy Bodies. Am. J. Geriatr. Psychiatry 25, 595-604.

Fiebelkorn, Ian C., Kastner, Sabine, 2019. The Puzzling Pulvinar. Neuron 101 (2), 201-203.

Frei, Karen, Truong, Daniel D., 2017. Hallucinations and the spectrum of psychosis in Parkinson's disease. J. Neurol. Sci. 374, 56-62.

Friston, K.J., Harrison, L., Penny, W., 2003. Dynamic causal modelling. Neuroimage 19 (4), 1273-1302.

Furl, N., 2015. Structural and effective connectivity reveals potential network-based influences on category-sensitive visual areas. Front. Hum. Neurosci. 9, 253.

Georgy, Loraine, Celeghin, Alessia, Marzi, Carlo A., Tamietto, Marco, Ptito, Alain, 2016. The superior colliculus is sensitive to gestalt-like stimulus configuration in hemispherectomy patients. Cortex 81, 151-161.

Goulden, Nia, McKie, Shane, Suckling, John, Williams, Stephen Ross, Anderson, Ian Muir, Deakin, John Francis William, Elliott, Rebecca, 2010. A comparison of permutation and parametric testing for between group effective connectivity differences using DCM. Neuroimage 50 (2), 509-515.

Grafton, Scot. T., Woods, Roger P., Tyszka, Mike, 1994. Functional imaging of procedural motor learning: Relating cerebral blood flow with individual subject performance. Hum. Brain Mapp. 1 (3), 221-234.

Guedy, C., Vuilleumier, P., 2020. Functional connectivity fingerprints of the human pulvinar: Decoding its role in cognition. Neuroimage 221, 1-11.

Hacker, C.D., Perlmutter, J.S., Criswell, S.R., Ances, B.M., Snyder, A.Z., 2012. Resting state functional connectivity of the striatum in Parkinson's disease. Brain 135, 3699-3711.

Harting, John K., Casagrande, V.A., Weber, Joseph T., 1978. The projection of the primate superior colliculus upon the dorsal lateral geniculate nucleus: autoradiographic demonstration of interlaminar distribution of tectogeniculate axons. Brain Res. 150 (3), 593-599.

Helmich, R.C., Derikx, L.C., Bakker, M., Scheeringa, R., Bloem, B.R., Toni, I., 2010. Spatial remapping of cortico-striatal connectivity in Parkinson's disease. Cereb Cortex 20, 1175-1186.

Hoehn, M.M., Yahr, M.D., 1967. Parkinsonism: onset, progression and mortality. Neurology 17, 427-442.

Kahan, Josh, Mancini, Laura, Urner, Maren, Friston, Karl, Hariz, Marwan, Holl, Etienne, White, Mark, Ruge, Diane, Jahanshahi, Marjan, Boertien, Tessel, Yousry, Tarek, Thornton, John S., Limousin, Patricia, Zrinzo, Ludvic, Foltynie, Tom, Baron, JeanClaude, 2012. Therapeutic subthalamic nucleus deep brain stimulation reverses cortico-thalamic coupling during voluntary movements in Parkinson's disease. PLoS ONE 7 (12), e50270. https://doi.org/10.1371/journal.pone.005027010.1371/ journal.pone.0050270.g00110.1371/journal.pone.0050270.g00210.1371/journal. pone.0050270.g00310.1371/journal.pone.0050270.g00410.1371/journal. pone.0050270.t00110.1371/journal.pone.0050270.t002.
Kahan, J., Urner, M., Moran, R., Flandin, G., Marreiros, A., Mancini, L., White, M., Thornton, J., Yousry, T., Zrinzo, L., Hariz, M., Limousin, P., Friston, K., Foltynie, T., 2014. Resting state functional MRI in Parkinson's disease: the impact of deep brain stimulation on 'effective' connectivity. Brain 137, 1130-1144.

Künzle, H., Akert, K., 1977. Efferent connections of cortical, area 8 (frontal eye field) in Macaca fascicularis. A reinvestigation using the autoradiographic technique. J. Comp. Neurol. 173 (1), 147-163.

Kuypers, Henricus G.J.M., Lawrence, Donald G., 1967. Cortical projections to the red nucleus and the brain stem in the Rhesus monkey. Brain Res. 4 (2-3), 151-188.

Lerner, Alicja, Bagic, Anto, 2008. Olfactory pathogenesis of idiopathic Parkinson disease revisited. Mov. Disord. 23 (8), 1076-1084.

Lock, T.M., Baizer, J.S., Bender, D.B., 2003. Distribution of corticotectal cells in macaque. Exp. Brain Res. 151 (4), 455-470.

Mahlknecht, Philipp, Seppi, Klaus, Poewe, Werner, 2015. The Concept of Prodromal Parkinson's Disease. J. Parkinsons Dis. 5 (4), 681-697.

Marreiros, André C., Cagnan, Hayriye, Moran, Rosalyn J., Friston, Karl J., Brown, Peter, 2013. Basal ganglia-cortical interactions in Parkinsonian patients. Neuroimage 66, 301-310.

Merigan, W.H., Maunsell, J.H., 1993. How parallel are the primate visual pathway? Annu. Rev. Neurosci. 16, 369-402.

Moro, Elena, Bellot, Emmanuelle, Meoni, Sara, Pelissier, Pierre, Hera, Ruxandra, Dojat, Michel, Coizet, Veronique, 2020. Visual dysfunction of the superior colliculus in de novo Parkinsonian patients. Ann. Neurol. 87 (4), 533-546.

Munoz, D.P., Coe, B.C., 2011. Saccade, search and orient-the neural control of saccadic eye movements. Eur. J. Neurosci. 33, 1945-1947.

Nackaerts, Evelien, Michely, Jochen, Heremans, Elke, Swinnen, Stephan, SmitsEngelsman, Bouwien, Vandenberghe, Wim, Grefkes, Christian, Nieuwboer, Alice, 2018. Being on Target: Visual Information during Writing Affects Effective Connectivity in Parkinson's Disease. Neuroscience 371, 484-494.

Najdenovska, Elena, Alemán-Gómez, Yasser, Battistella, Giovanni, Descoteaux, Maxime, Hagmann, Patric, Jacquemont, Sebastien, Maeder, Philippe, Thiran, Jean-Philippe, Fornari, Eleonora, Bach Cuadra, Meritxell, 2018. In-vivo probabilistic atlas of human thalamic nuclei based on diffusion- weighted magnetic resonance imaging. Sci. Data 5 (1). https://doi.org/10.1038/sdata.2018.270.

Nettersheim, Felix Sebastian, Loehrer, Philipp Alexander, Weber, Immo, Jung, Fabienne, Dembek, Till Anselm, Pelzer, Esther Annegret, Dafsari, Haidar Salimi, Huber, Carlo Andreas, Tittgemeyer, Marc, Timmermann, Lars, 2019. Dopamine substitution alters effective connectivity of cortical prefrontal, premotor, and motor regions during complex bimanual finger movements in Parkinson's disease. Neuroimage 190, $118-132$.

Nicolas, G., Castet, E., Rabier, A., Kristensen, E., Dojat, M., Guerin-Dugue, A., 2021. Neural Correlates of Intra-saccadic Motion Perception. J. Vision 21, 1-24.

Palmer, S.J., Eigenraam, L., Hoque, T., McCaig, R.G., Troiano, A., McKeown, M.J., 2009. Levodopa-sensitive, dynamic changes in effective connectivity during simultaneous movements in Parkinson's disease. Neuroscience 158 (2), 693-704.

Pennartz, Cyriel M.A., Dora, Shirin, Muckli, Lars, Lorteije, Jeannette A.M., 2019. Towards a unified view on pathways and functions of neural recurrent processing. Trends Neurosci. 42 (9), 589-603.

Penny, Will D., Stephan, Klaas E., Daunizeau, Jean, Rosa, Maria J., Friston, Karl J., Schofield, Thomas M., Leff, Alex P., Kording, Konrad P., 2010. Comparing families of dynamic causal models. PLoS Comput. Biol. 6 (3), e1000709. https://doi.org/ 10.1371/journal.pcbi.100070910.1371/journal.pcbi.1000709.g00110.1371/ journal.pcbi.1000709.g00210.1371/journal.pcbi.1000709.g00310.1371/journal. pcbi.1000709.g00410.1371/journal.pcbi.1000709.g00510.1371/journal. pcbi.1000709.g00610.1371/journal.pcbi.1000709.g00710.1371/journal. pcbi.1000709.g00810.1371/journal.pcbi.1000709.t00110.1371/journal. pcbi.1000709.t00210.1371/journal.pcbi.1000709.s001.

Pereira, Joana B., Junqué, Carme, Martí, María-Jośe, Ramirez-Ruiz, Blanca, Bargalló, Nuria, Tolosa, Eduardo, 2009. Neuroanatomical substrate of visuospatial and visuoperceptual impairment in Parkinson's disease. Mov. Disord. 24 (8), 1193-1199.

Postuma, Ronald B., Berg, Daniela, Stern, Matthew, Poewe, Werner, Olanow, C. Warren, Oertel, Wolfgang, Obeso, José, Marek, Kenneth, Litvan, Irene, Lang, Anthony E., Halliday, Glenda, Goetz, Christopher G., Gasser, Thomas, Dubois, Bruno, Chan, Piu, Bloem, Bastiaan R., Adler, Charles H., Deuschl, Günther, 2015. MDS clinical diagnostic criteria for Parkinson's disease. Mov. Disord. 30 (12), 1591-1601.

Rolland, M., Carcenac, C., Overton, P.G., Savasta, M., Coizet, V., 2013. Enhanced visual responses in the superior colliculus and subthalamic nucleus in an animal model of Parkinson's disease. Neuroscience 252, 277-288.

Rowe, J., Stephan, K.E., Friston, K., Frackowiak, R., Lees, A., Passingham, R., 2002. Attention to action in Parkinson's disease: impaired effective connectivity among frontal cortical regions. Brain 125, 276-289.

Rowe, J.B., 2010. Connectivity analysis is essential to understand neurological disorders. Front. Syst. Neurosci. 4.

Rowe, J.B., Hughes, L.E., Barker, R.A., Owen, A.M., 2010. Dynamic causal modelling of effective connectivity from fMRI: are results reproducible and sensitive to Parkinson's disease and its treatment? Neuroimage 52 (3), 1015-1026.

Schneider, Keith A., Kastner, Sabine, 2005. Visual responses of the human superior colliculus: a high-resolution functional magnetic resonance imaging study. J. Neurophysiol. 94 (4), 2491-2503.

Sherman, S.M., Koch, C., 1986. The control of retinogeniculate transmission in the mammalian lateral geniculate nucleus. Exp. Brain Res. 63, 1-20.

Siderowf, A., Jennings, D., Eberly, S., Oakes, D., Hawkins, K.A., Ascherio, A., Stern, M.B., Marek, K., Investigators, P., 2012. Impaired olfaction and other prodromal features in the Parkinson At-Risk Syndrome Study. Mov. Disord. 27, 406-412. 
Siderowf, A., Lang, A.E., 2012. Premotor Parkinson's disease: concepts and definitions. Mov. Disord. 27, 608-616.

Skidmore, F., Korenkevych, D., Liu, Y., He, G., Bullmore, E., Pardalos, P.M., 2011. Connectivity brain networks based on wavelet correlation analysis in Parkinson fMRI data. Neurosci. Lett. 499, 47-51.

Sonninen, T.M., Hamalainen, R.H., Koskuvi, M., Oksanen, M., Shakirzyanova, A., Wojciechowski, S., Puttonen, K., Naumenko, N., Goldsteins, G., Laham-Karam, N., Lehtonen, M., Tavi, P., Koistinaho, J., Lehtonen, S., 2020. Metabolic alterations in Parkinson's disease astrocytes. Sci. Rep. 10, 14474.

Stephan, K.E., Penny, W.D., Moran, R.J., den Ouden, H.E., Daunizeau, J., Friston, K.J., 2010. Ten simple rules for dynamic causal modeling. Neuroimage 49, 3099-3109. van Eimeren, T., Monchi, O., Ballanger, B., Strafella, A.P., 2009. Dysfunction of the default mode network in Parkinson disease: a functional magnetic resonance imaging study. Arch. Neurol. 66, 877-883.

Weil, R.S., Schrag, A.E., Warren, J.D., Crutch, S.J., Lees, A.J., Morris, H.R., 2016. Visual dysfunction in Parkinson's disease. Brain 139, 2827-2843.

Weller, R.E., Steele, G.E., Kaas, J.H., 2002. Pulvinar and other subcortical connections of dorsolateral visual cortex in monkeys. J. Comp. Neurol. 450, 215-240.

Wu, T., Wang, L., Chen, Y., Zhao, C., Li, K., Chan, P., 2009. Changes of functional connectivity of the motor network in the resting state in Parkinson's disease. Neurosci. Lett. 460, 6-10.

Wurtz, R.H., Goldberg, M.E., 1972. The role of the superior colliculus in visually-evoked eye movements. Bibl. Ophthalmol. 82, 149-158. 